\title{
Opiate withdrawal syndrome in buprenorphine abusers admitted to a rehabilitation center in Tunisia.
}

\author{
Ines Derbel ${ }^{1}$, Asma Ghorbel ${ }^{1,2,3}$, Férièle Messadi Akrout ${ }^{1,2}$, Abdelmajid Zahaf ${ }^{4}$
}

1. Faculty of Pharmacy, University of Monastir, Tunisia

2. Hygiene Laboratory Hedi Chaker Hospital, South of Tunisia

3. Research Laboratory Toxicology and Environment RL12SP07

4. Tunisian Association for the Prevention of Drug Use

\begin{abstract}
Background: Illicit use of high dosage buprenorphine has been well documented in several countries, including Tunisia.

Objectives: The aim of this survey is to assess the buprenorphine withdrawal syndrome time course, and how it may be affected by the population characteristics among subjects admitted to a rehabilitation center in Tunisia.

Methods: A prospective research has permitted study of the socio-demographic characteristics and assessment of buprenorphine withdrawal syndrome among 32 subjects admitted for buprenorphine dependence by using the clinical opiate withdrawal scale. An ANOVA was conducted to examine the effect of different factors on the withdrawal scores.

Results: 32 subjects were included. Among them 30 were males, 27 had been injecting buprenorphine, 16 were poly-drug abusers and 2 had a history of mental disorders. Buprenorphine withdrawal syndrome was of a mild intensity and had a delayed onset. Withdrawal mean scores varied between 0 and 9 , and maximum values were reached at day 21 . These scores varied significantly over time $(\mathrm{p}<0,001)$. The sex $\mathrm{v}$ time interaction and the mode of consumption of buprenorphine had significant effects on the withdrawal scores $(p<0,001)$. The poly-drug consumption and the history of mental disorders did not have any significant effect on the withdrawal scores.

Conclusion: This study has permitted description of buprenorphine withdrawal syndrome among patients going through a detoxification treatment at a rehabilitation center. Understanding this syndrome would help elaborate effective and suitable buprenorphine dependence management plans.

Keywords: Buprenorphine, dependence, withdrawal.

DOI: http://dx.doi.org/10.4314/ahs.v16i4.24

Cite as: Derbel I, Ghorbel A, Akrout FM, Zahaf A. Opiate withdrawal Syndrome in buprenorphine abusers admitted to a rehabilitation center in Tunisia. Afri Health Sci. 2016;16(4): 1067-1077. http://dx.doi.org/10.4314/abs.v16i4.24
\end{abstract}

\section{Introduction}

Buprenorphine is a semi-synthetic opioid derived from thebaine, discovered in the early $1970 \mathrm{~s}^{1}$. It was firstly marketed for parenteral use at low doses of $0.3 \mathrm{mg} / \mathrm{mL}$ in 1978 in the United-Kingdom, under the name Temgésic ${ }^{\circledR}$. At that time it was used as an analgesic for post-operative and cancer patients. Ever since, buprenorphine has generated a lot of interest in the treatment of opioid dependence and its effectiveness for this has been proven at high doses ${ }^{2}$. Later,in 1995, France was the first country to
Corresponding author:
Ines Derbel,
Faculty of Pharmacy,
University of Monastir, Tunisia
Email: ines.drbl@gmail.com

market high-dosage buprenorphine (HDB), as Subutex ${ }^{\circledR}$, for opioid maintenance therapy. Subutex was available in sublingual tablets formulated at $0.4 \mathrm{mg}, 2.0 \mathrm{mg}$ and 8.0 mg doses.

Though buprenorphine has provided a major advance in opioid dependence treatment, as it is so widespread, cases of abuse and diversion have been widely documented . In the Middle East and North Africa (MENA), opioid substitution treatment is only available in 5 out of the 20 countries, yet, HDB is one of the most abused drugs by People Who Inject Drugs (PWID) in the region ${ }^{5}$.

In Tunisia, Subutex ${ }^{\circledR}$ does not have a license to be marketed and, hence, is not used in medical practice. However, it is smuggled across borders and is widely trafficked for misuse. According to some Non-Governmental Or- 
ganizations (NGO) reports, the estimated number of PWID was around 9000 in $2011^{5}$, and HBD was the most abused drug. Even though these numbers are growing from year to year, there are no legal specifications related to substance abuse treatments or opioid substitution therapies. The only residential rehabilitation program available in the country has been provided in a center run by the Tunisian Association for the Prevention of Drug Use "ATUPRET".

In 2013, half of the center's admissions were related to HDB diversion and abuse ${ }^{7}$. For buprenorphine abusers, ATUPRET center offers medical care services throughout the spontaneous drug withdrawal process. This is why the understanding of the buprenorphine withdrawal syndrome is needed for a proper medical management. Previous studies that have described this syndrome were either laboratory-based, conducted on small samples, or included subjects that were not necessarily buprenorphine dependent .

This study was conducted in order to assess the opiate withdrawal syndrome in buprenorphine dependent patients. The syndrome's time course evolution was described and analyzed in accordance with the population characteristics.

\section{Methods}

\section{Study context and sample}

The present study followed buprenorphine withdrawal syndrome in subjects who sought treatment for buprenorphine dependence from the ATUPRET rehabilitation center between February and May 2015. The center has only provided its services to male subjects. However, as a form of a trial led in April 2015, it allowed 10 female patients suffering from different types of dependence to be admitted.

Subjects recruited for this study were those who had completed three out of the four-week treatment protocol specified by the center. Thirty males and two female patients met these criteria and were, thus, included in the present study.

\section{Data collection}

\section{Socio-demographic characteristics}

Socio-demographic data was collected using a form that was conceived after analyzing the medical and the social records of other patients admitted to the center. The form included items pertaining to each patient's de- mographics, medical history, other substance abuse and addictive behavior. Permission was given to visit the rehabilitation center twice a week and ethical approval was obtained from the center's board.

\section{Withdrawal score}

The buprenorphine withdrawal syndrome was assessed using the Clinical Opiate Withdrawal Scale (COWS). It consists of 11 observed (clinician-rated) and subjective (patient-rated) items. The score attributed to each item reflects the severity of the symptom. The following cutscores for the COWS have been offered: $5-12=$ mild, $13-$ $24=$ moderate, $25-36$ moderately severe, and $>36$ severe withdrawal ${ }^{11}$.

\section{Measures}

COWS score was measured twice a week, during the patient's stay in the center. The measures were named V1, V2, V3, V4, V5, V6, V7 and V8 corresponding respectively to days $3,7,10,14,17,21,24$ and 28 of the patient's stay. The score was then calculated according to the observed withdrawal signs as well as those reported by the patient during the interview.

All the data was collected anonymously and after the subject's consent.

\section{Data analysis}

The data was collected and analyzed using IBM SPSS and Microsoft Excel 2013. Descriptive statistics were produced according to the demographic characteristics and withdrawal scores.

Peak scores on the COWS were determined for each measure, and data was analyzed using repeated-measures one factor Analysis of Variance (ANOVA). Time course differences according to the population characteristics (gender, means of buprenorphine intake, history of mental disorder and nature of the addiction) were examined using repeated measures mixed model ANOVA (characteristic, time and characteristic $\times$ time). The degrees of freedom were adjusted using Greenhouse-Geisser corrections for violation of the sphericity assumption. Pairwise comparisons for significant main effects were examined using Bonferroni adjustment. Statistical significance was indicated when p-value $<0.05$.

\section{Results \\ Population characteristics}

A total of 32 individuals were included, 30 were male and

African Health Sciences Vol 16 Issue 4, December, 2016 
2 female. The mean age was $38.3 \pm 9.8$ years (Table 1). They were mostly unmarried $(n=21)$, only 17 were employed, and 14 reported elementary school or lower level of education. Infection with the Hepatitis $C$ virus was found in $53.1 \%$ of the patients, $3.1 \%$ were $\mathrm{HBV}$ positive and $18.6 \%$ were HIV positive. The presence of mental disorders was reported by 2 subjects, without any specifications regarding the disorder itself. All subjects were smokers and 16 were abusing different substances other than buprenorphine (Table 1).

Table 1 : sociodemographic characteristics

\begin{tabular}{lll}
\hline & Subjects & Missing (\%) \\
\hline Sex (males), n(\%) & $30(93.8 \%)$ & 0 \\
Mean Age (SD) & $38.34(9.81)$ & 0 \\
Nationality (Tunisian), n (\%) & $20(68.8)$ & 0 \\
Marital status (married), n (\%) & $11(34.4)$ & 0 \\
Homeless, n(\%) & $2(6.3)$ & 25 \\
Serology (known), n(\%) & $24(75)$ & 0 \\
Hepatitis C (positive), n(\%) & $17(53.1)$ & 0 \\
Hepatitis B (positive), n(\%) & $1(3.1)$ & 0 \\
HIV (positive), n(\%) & $6(18.6)$ & 15.6 \\
Education (elementary school or less), n(\%) & $14(51.9)$ & 3.1 \\
Employed, n(\%) & $17(53.1)$ & 6.3 \\
History of mental disorders, n(\%) & $2(6.25)$ & 0 \\
Poly-drug abuser, n(\%) & $16(53.3)$ & 0 \\
\multicolumn{1}{c}{ Cannabis } & $11(36.7)$ & 0 \\
$\quad$ Alcohol $\quad$ Lorazepam & $16(53.3)$ & 0 \\
Tobacco & $5(15.6)$ & 0 \\
IV user, n(\%) & $32(100)$ & \\
\multicolumn{1}{c}{ HIV : Human Immunodeficiency Virus } & $27(84.4)$ &
\end{tabular}

\section{Buprenorphine abuse pattern}

The majority $(n=27)$ reported intravenous intake and the daily intake varied from 1 to $10 \mathrm{mg}$, the mean dose was $6.22 \mathrm{mg}(\mathrm{SD}=3.6)$. The mean duration of buprenorphine abuse was 11.7 years $(\mathrm{SD}=7.79)$.

\section{Withdrawal scores}

Withdrawal scores on the COWS varied between 0 and 9. The mean score increased progressively from an initial value of $3.40(\mathrm{SD}=1.58)$ on $\mathrm{V} 1$ to reach a maximum value of $6.1(\mathrm{SD}=1.47)$ on $\mathrm{V} 5$ then decreased during the last week, to a value of $1.97(\mathrm{SD}=.92)$ by V8. Before V4, all scores were $<5$ (Figure1).

On repeated measure ANOVA a significant effect of time was observed $(\mathrm{F}=51.2, \mathrm{p}<0,001)$. Significant differences on Bonferroni comparisons of COWS ratings were found on V4 and V7 (scores on V4 being higher).

Withdrawal scores varied differently upon the population's characteristics. The Table 2 summarizes statistical results from the repeated measures mixed model ANOVA for the different effects of characteristics and interactions on COWS ratings. 
Table 2: Statistical summary of the ANOVA analysis

\begin{tabular}{|c|c|c|}
\hline Measure & $\mathbf{F}$ & $p$-value \\
\hline gender $\times$ time & $\mathrm{F}(4.24,127.3)=2.89$ & 0.023 \\
\hline Means of intake $\times$ time & $F(4.11,123.3)=0.565$ & 0.693 \\
\hline $\begin{array}{l}\text { History of psychaitric } \\
\text { disorder } \times \text { time }\end{array}$ & $\mathrm{F}(4.26,110.9)=11.02$ & 0.348 \\
\hline Nature of dependance $\times$ time & $F(3.97,111.1)=0.58$ & 0.676 \\
\hline
\end{tabular}

Interactions for which $\mathrm{p}<0.05$ are considered significant

\section{Effect of the gender on withdrawal ratings}

The ANOVA revealed a significant effect of the interaction gender $\times$ time on the withdrawal scores. Thus, scores on the COWS depended upon gender and time. The analysis of the main simple effects revealed a significant effect of time on withdrawal scores for both men $(\mathrm{F}=65.4$, $\mathrm{p}<0.001)$ and women $(\mathrm{F}=10.8, \mathrm{p}<0.001)$. A significant effect of gender on withdrawal scores was been found on V1 $(\mathrm{F}=6.8, \mathrm{p}=0.014)$ and V2 $(\mathrm{F}=6.8, \mathrm{p}=0.007)$ and the scores were higher for the female patients. For the rest of the withdrawal period, no significant difference was found.
Bonferroni multiple comparisons were used to compare the withdrawal time course for each gender. For women, the initial score on V1 $(M=6 \pm 0.0)$ was only significantly different than the score measured on V8 $(M=1.5 \pm 0.707)$ $\mathrm{p}=0.014$. However, men's scores increased significantly from V1 $(\mathrm{M}=3.23 \pm 1.48)$ to V3 $(\mathrm{M}=4.27 \pm 1.31, \mathrm{p}=0.006)$ and V4 $(\mathrm{M}=5.57 \pm 1.59, \mathrm{p}=0.001)$ then decreased significantly on $\mathrm{V} 7(\mathrm{M}=3.32 \pm 1.21, \mathrm{p}<0.001)$ and $\mathrm{V} 8$ (M1.99 $\pm 0.91, \mathrm{p}<0.001)$.

Therefore, withdrawal was significantly more intense for woman during the first week (V1 and V2). For each gender, scores did not vary in the same way as withdrawal ratings for men varied more frequently during the treatment period (Figure1). 


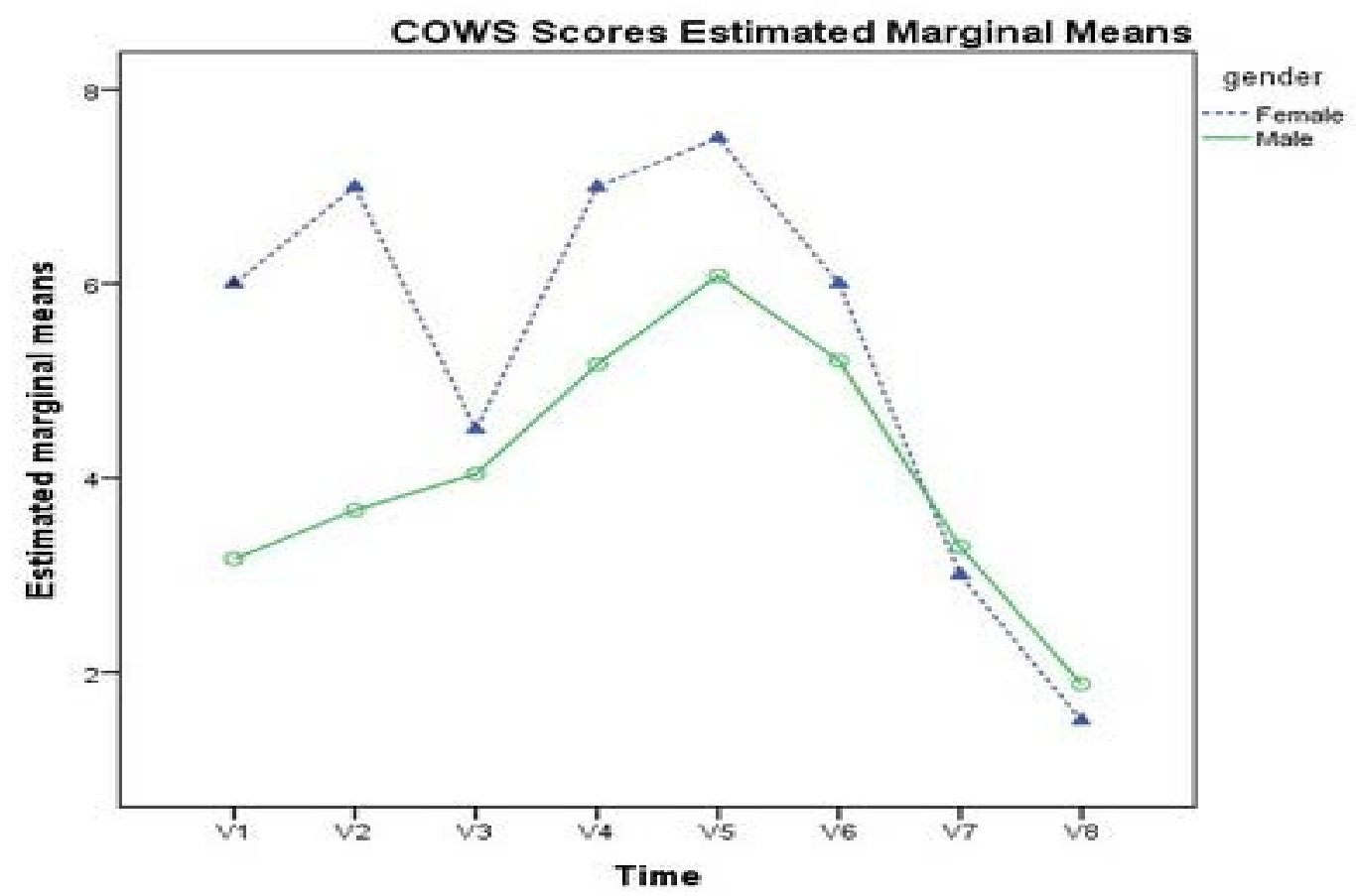

Figure 1: Evolution of withdrawal scores by patient's gender; Women withdrawal scores, measured using the COWS were, overall, higher than men scores. The intersection between the two plots indicates a significant effect of the interaction gender $\times$ time $\backslash r \backslash n$

Effect of the means of intake on withdrawal ratings The analysis of data did not find a significant effect of the interaction means of intake $x$ time on COWS ratings $(F=0.565, p=0,693)$. However, it revealed significant main effects of the means of intake $(\mathrm{F}=7.47, \mathrm{p}=0.01)$ and time $(\mathrm{F}=22.41, \mathrm{p}<0.001)$ on the withdrawal scores.
Withdrawal scores measured for the individuals who were injecting buprenorphine $(M=4,45 \pm 0,155)$ were significantly higher compared to those who were snorting the drug $(M=3.38 \pm 0.36)$. The figure 2 shows that, even though the injector group had higher COWS scores, the withdrawal plots of the two groups varied in the same way without any intersections. 


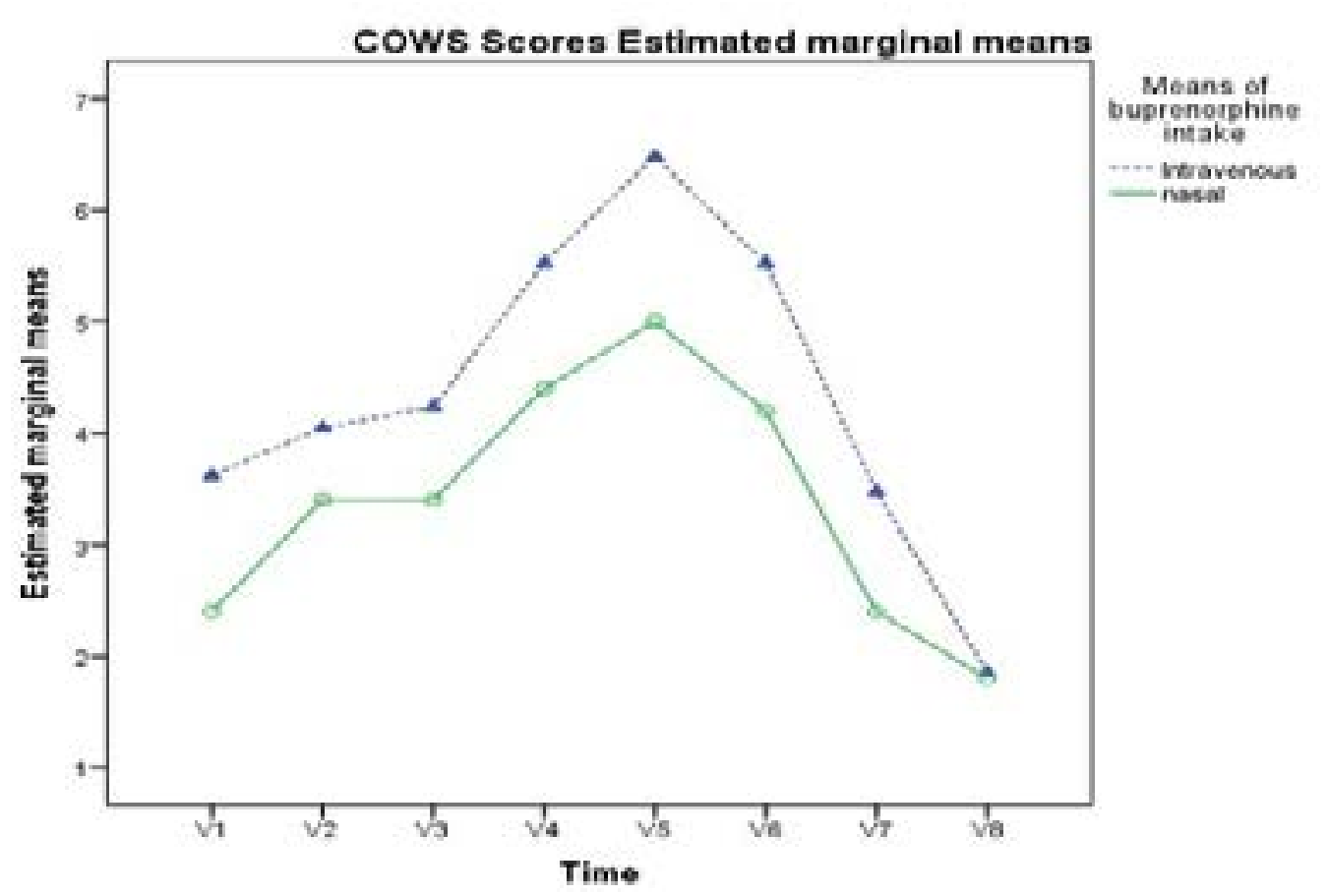

Figure 2: Evolution of withdrawal scores by the route of buprenorphine abuse

Effect of the history of the mental disorders on withdrawal ratings

The population was divided into two groups according to the history of mental disorders. The effect of the in- teraction of the history of mental disorders $\times$ time was not significant, and neither was the effect of the history of mental disorders alone. Only time was found to have a significant effect $(\mathrm{F}=11.02, \mathrm{p}<0,001)$ on the withdrawal scores within the two groups. (Figure3)

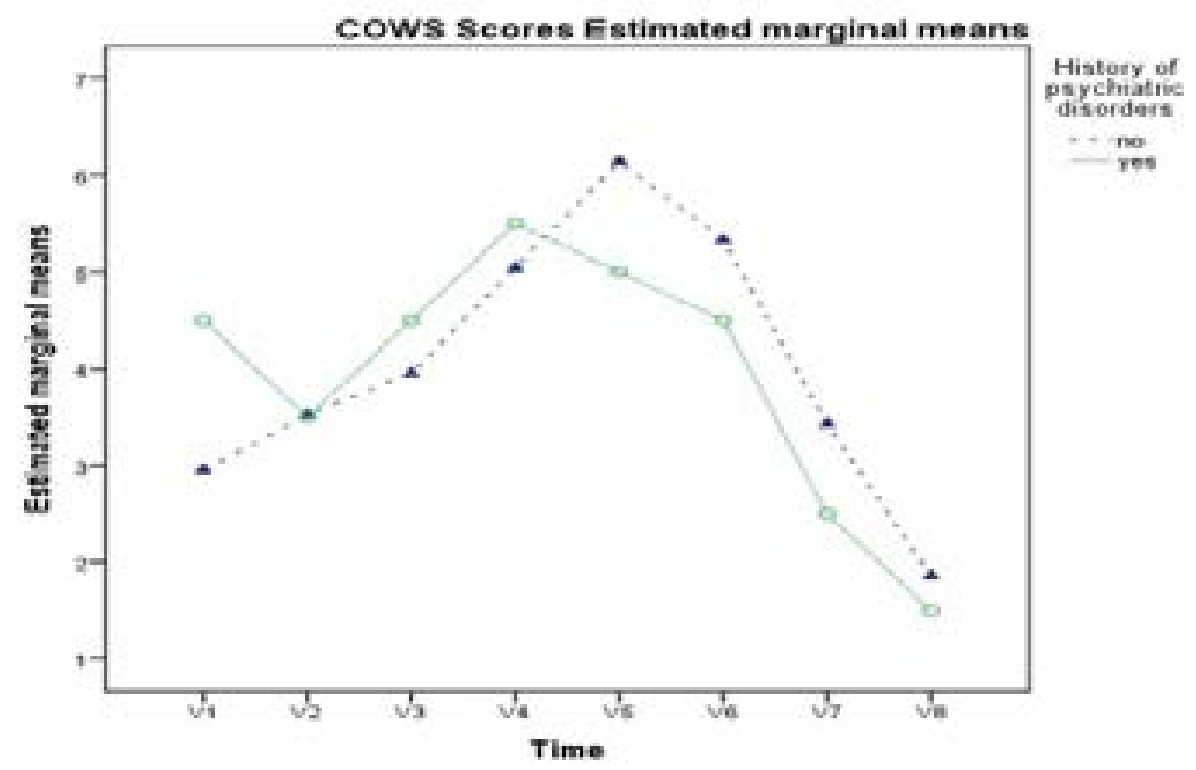

Figure 3: Evolution of withdrawal scores according to patients' psychiatric history; Withdrawal scores were measured using The COWS for patients with a history of a mental disorder and those who had not reported any. No significant difference was found between the two groups; 


\section{Withdrawal management}

Withdrawal management in the center was mainly symptomatic. Medications used were from the therapeutic classes: non-opioid analgesics, non-steroidal anti-inflammatory drugs, antispasmodics, antihistamines and benzodiazepines.

\section{Discussion}

The main findings of this study allowed assessment of the evolution and the characteristics of the opioid withdrawal syndrome in subjects with buprenorphine dependence without a maintenance treatment using the COWS. Most of these demographic characteristics of the 32 individuals included are consistent with findings from previous studies describing the Tunisian buprenorphine abuser profile ${ }^{7,12}$. Subjects were mainly men, unmarried and almost half of them were unemployed. Celibacy, unemployment and low levels of education have also been associated to buprenorphine abuse in other countries such as Canada and Finland ${ }^{13,14}$.

Buprenorphine was abused mainly by IV route $(84.4 \%)$ which may explain the high prevalence of Hepatitis $C$ virus (HCV) (53.1\%), Hepatitis B (3.1\%) virus (HBV) and HIV $(18.6 \%)$ infection. These results are similar to the results found in a study led in 2007 by Kilani B et al. evaluating the concomitant infection by HCV and HIV in Tunisia. It was found that $39.7 \%$ of the HIV- positive included patients were also HCV-positive and that most of them $(78.4 \%)$ had a history of drug injection ${ }^{7}$. Thus, those results along with the findings of this current study highlight the burden of the rapid expansion of HIV and HCV coinfection in PWID in Tunisia.

Buprenorphine withdrawal syndrome assessed in the population had a delayed appearance and a mild intensity. In fact, according to the COWS, scores have to be $\geq 5$ in order to indicate the presence of opioid withdrawal, while during the first two weeks of the study, all ratings were $<5$. A mild withdrawal was measured from V4 (day14) to V6 (day 21). These results can be explained by buprenorphine's pharmacokinetics and pharmacodynamics. The compound is lipophilic. It shows a high volume of distribution and distributes well in tissues including the brain. Buprenorphine has a long elimination half-life, is highly protein- bound $(96 \%)$ and has a great affinity to $\mu$ opioid receptors ${ }^{2,15}$. In addition, buprenorphine undergoes glucuronidation into buprenorphine-3- glucuronide and it seems that this metabolite may be reabsorbed as buprenorphine after entero-hepatic recirculation ${ }^{16}$. The combination of these properties may have contributed to prolonging the effect of buprenorphine, after the last intake, enough to dissimulate withdrawal signs during the first 14 days of the process. The medication also slowly dissociates from $\mu$ receptors ${ }^{1}$. While studying the effect of buprenorphine on $\mu$ receptors on heroin dependent individuals, Greenwald et al stated that the appearance of withdrawal signs was highly correlated to the buprenorphine $\mu$ receptors occupancy rates. They also found that almost $20 \%$ of the receptors were occupied by the molecule 76 hours after the last buprenorphine's administration $^{17}$. Therefore, buprenorphine slow rates of dissociation from $\mu$ receptors might have allowed the return of cellular homeostasis in the parts of the central nervous system (CNS) thought to be responsible for opioid withdrawal resulting, thereby, in a mild withdrawal syndrome.

Withdrawal scores measured for female patients were higher than those measured for males. The interaction gender $\times$ time was significant indicating that the time course of withdrawal did not follow the same schema for the two genders.

Two main factors may explain the gender-related difference noted in the withdrawal ratings. The first one is due to the innate difference between men and women in the somatic and visceral perception. In order to measure withdrawal score, the COWS evaluates the levels of bone and joint aches as well as gastro intestinal cramps ${ }^{11}$. However, males and females may label and describe the same noxious sensation differently. It has also been found that pain threshold for women was lower. On a biological level, the effect that sexual hormones have on the CNS and the menstrual cycle was shown to influence sensitivity to pain $^{18}$. Zubieta et al, have been examining $\mu$ receptors binding differences between men and women, they found that women showed more $\mu$-opioid receptor availability than men in several brain regions, meaning that anti-nociception and reward systems may be modulated differently according to the gender.

The second reason is related to buprenorphine's pharmacokinetics. Moody D.E et al stated that after the administration of equivalent doses of buprenorphine, the compound did not show the same kinetics for the two sexes. Females were exposed to higher levels of buprenorphine and its metabolites. In normal therapy, this may be non-critical ${ }^{19}$. However, it could be taken into 
consideration when dealing with cases of buprenorphine dependence and abuse.

For now, the issues of the gender differences in buprenorphine withdrawal perception are not known. Long-term follow-up is necessary in order to understand if these facts may influence treatment outcome or be associated to relapses.

Withdrawal scores for buprenorphine injectors $(84.4 \%$ of the population), were significantly higher compared to patients who were abusing it by nasal route. The effect of the interaction means of intake $\times$ time was not significant. This result was expected since $\mu$-opioid receptors occupancy rates depend on buprenorphine plasma concentration, and thus, bioavailability which varies according to the route of administration. When used intravenously, buprenorphine bioavailability is $100 \%$ versus $50 \%$ when it is snorted ${ }^{7}$. Furthermore, the amount of drug wasted would be greater after nasal intake.

Two patients reported that they had suffered from a mental disorder before they were admitted to the rehabilitation center and the nature of the disorder was not specified. Furthermore, the lack of data related to psychiatric counseling in the medical records did not allow determination as to whether or not other cases were diagnosed during their stay in the center.

The relationship between psychiatric disorders and substance use disorders is complex ${ }^{22}$.

When we compared withdrawal in subjects suffering from psychiatric disorders with the rest of the population, no significant difference between the two groups was found. However, these results cannot be generalized since in the present study the nature of the mental disorder was not specified. The outcomes of the association between psychiatric troubles and substance use disorder may vary depending on the trouble and the substance involved ${ }^{23,24}$. Thereby, an initial diagnosis of psychiatric disorders before admission to the detoxification process may be mandatory for the proper management of withdrawal signs and the adequate adaptation of the treatment, preventing the exacerbation of underlying psychiatric co-morbidities $^{23}$.

We found that buprenorphine abuse was associated with the abuse of other psychoactive substances in 16 individuals. Alcohol, cannabis, and Lorazepam were the most frequently abused.

The use of cannabis and benzodiazepines is frequent among prescription opioids and buprenorphine users .Nevertheless, some of these associations can be more dangerous than others and buprenorphine may become lethal when associated with alcohol and/ or benzodiazepines. It is known that CYP3A4 inducers and inhibitors may reduce or improve buprenorphine $\mathrm{N}$-alkylation. However, it appears that the interaction between buprenorphine and benzodiazepines or alcohol is more likely to be of a pharmacodynamic rather than of a pharmacokinetic nature ${ }^{26,27}$. The ceiling effect on the respiratory function of buprenorphine may not be present when it is co-administered with other psychoactive drugs ${ }^{27}$. In fact, CNS depressants, particularly benzodiazepines, act synergistically with opioids to reduce the respiratory function, which increases the toxicity of opioids ${ }^{4,26}$. Therefore, since buprenorphine has very strong $\mu$-receptor affinity, naloxone in standard doses does not reverse the effects of buprenorphine and cannot be used in case of respiratory depression and higher doses must be used ${ }^{15,28}$. Poly-drug abusers had higher withdrawal scores compared to individuals abusing buprenorphine alone. This difference was, however, non-significant $(p=0,172)$.

In a general manner, non-opioid drugs have little proved efficiency on opioid withdrawal signs and only benzodiazepines have been reported to use a moderate improve on the withdrawal ${ }^{29}$. Nonetheless, the prolonged use exposes the patient to the risk of developing a co-dependence to benzodiazepines ${ }^{30}$. This co-dependence may worsenthe opiate withdrawal. Benzodiazepine withdrawal may lead to a reduced GABA-ergic and an increased glutamatergic activity in the locus coeruleus. When this occurs concurrent with opiate withdrawal, it may enhance noradrenaline release, andthereby exacerbate opiate withdrawal ${ }^{31}$. In this study, most patients were abusing more than two substances concomitantly, making it difficult to evaluate the effect of each substance apart.

These results highlight the importance of the establishment of an adapted treatment for each patient according to his dependence and drug abuse patterns.

The management of the withdrawal syndrome in the center was symptomatic. Drugs were administered upon patients' request and the clinical judgement of nurses to help manage withdrawal symptoms. The most effective pharmacotherapies for opioid withdrawal are substitution therapies. The approach involves using $\mu$-opioid agonists with long half-lives, such as buprenorphine and methadone that will reduce withdrawal symptoms and produce 
less drug-like effects. Up to the present time, there has been no evidence-based guidelines for the management of buprenorphine injection ${ }^{32}$. In a trial led in Iran by Ahmadi and Ahmadi K, 204 buprenorphine injectors were randomized to receive methadone(orally), buprenorphine (sublingually) or naltrexone, an opioid antagonist, (orally) along with counselling sessions. By the end of the treatment period, results were in favor of methadone ${ }^{33}$. However, these results need more investigation before they can be generalized ${ }^{32}$.

Some countries like the United-States ${ }^{2}$, France ${ }^{34}$ or Malay$\mathrm{sia}^{32}$, use Suboxone ${ }^{\circledR}$, an association of buprenorphine and naloxone, as part of their dependence management plan. On terms of effectiveness, this combination can be compared to Subutex ${ }^{\circledR}$ in the treatment of opioid dependence, as naloxone is not absorbed sublingually. However, since it can be absorbed via the IV and the nasal routes, Suboxone ${ }^{\circledR}$ may precipitate withdrawal and help, hence, reduce buprenorphine misuse ${ }^{34}$. In 2006, the Malaysian government, replaced buprenorphine with the combination buprenorphine/ naloxone to address the buprenorphine diversion concern. After this transition, no reduction in injection related risk behaviors was stated. On the contrary, it was found that there was an increase in the consumption of benzodiazepines and buprenorphine and patients have developed new methods to avoid the naloxone effects ${ }^{32}$. Giving the specificity of the buprenorphine abuse issue, more comparative studies focusing on the management of the buprenorphine withdrawal syndrome, should be conducted in the future to establish an effective treatment protocol.buprenorphine misuse ${ }^{34}$. In 2006, the Malaysian government, replaced buprenorphine with the combination buprenorphine/ naloxone to address the buprenorphine diversion concern. After this transition, no reduction in injection related risk behaviors was stated. Onthe contrary, it was found that there was an increase in the consumption of benzodiazepines and buprenorphine and patients have developed new methods to avoid the naloxone effects ${ }^{32}$. Giving the specificity of the buprenorphine abuse issue, more comparative studies focusing on the management of the buprenorphine withdrawal syndrome, should be conducted in the future to establish an effective treatment protocol.

In Tunisia, substitution treatments do not have a license to be marketed and are not legally available. This draconian approach does not take into account the benefits of

African Health Sciences Vol 16 Issue 4, December, 2016 the use of such medications on the health care system, especially when it comes to providing efficient treatments for opioid dependence. Besides, it seems that throughout the past years, this approach was not likely to put an end entirely to the diversion and the misuse of buprenorphine. According to a recent report of the WHO Expert Committee on Drug Dependence, despite the problematic increase of the buprenorphine misuse, its risk-benefit balance remains favorable.

\section{Limitations}

Some limitations need to be acknowledged. Withdrawal assessment could not be done on a daily basis. In any case, the sample was divided into subgroups of non-homogenous sizes and the subjects were not abusing the same amounts of buprenorphine before getting into the center. Data related to daily buprenorphine intake were based on client's self reporting and, therefore, may be subject to recall bias.

\section{Conclusion}

This study has permitted the description of the buprenorphine withdrawal syndrome. Given the fact that the withdrawal had a mild intensity and a delayed onset, symptoms might be mistakenly attributed to some other cause by both practitioners and patients. Adapted treatment protocols should be investigated and provided to reduce risky behaviors. Future studies should focus on long-term follow-ups and evaluate the efficiency of the access to opioid substitution treatment on this kind of dependence.

\section{Acknowledgements}

The authors sincerely thank the nursing staff working at the ATUPRET rehabilitation center, who helped immensely in this research.

\section{Conflict of interest}

None to declare

\section{References}

1. Khanna IK, Pillarisetti S. Buprenorphine-an attractive opioid with underutilized potential in treatment of chronic pain. Journal of pain research. 2015;8:859.

2. Yokell MA, Zaller ND, Green TC, Rich JD. Buprenorphine and buprenorphine/naloxone diversion, misuse, and illicit use: an international review. Current drug abuse reviews. 2011;4(1):28. 
3. ROUSSELLE Claire. Gestion à l'officine de la buprénorphine en tant que médicament de substitution aux opiacés. Bénéfices apportés, limites et évolution de la législation. [Thesis]. Nancy: Université Henri Poincaré, UFR Sciences pharmaceutiques et biologiques; 2009.

4. Uosukainen H, Kauhanen J, Bell JS, Ronkainen K, Tiihonen J, Fohr J, et al. Mortality among clients seeking treatment for buprenorphine abuse in Finland. Drug and alcobol dependence. 2013;133(2):391-7.

5. Rahimi-Movaghar A, Amin-Esmaili M, Aaraj E, Hermes J. Assessement of situation and response of drug use and its harms in the Middle East and North Africa 2012 [updated 2016/05/03/16:12:09]. Available from: http:// www.menahra.org/images/pdf/Menahra.pdf.

6. Aounallah-Skhiri H, ZALILA H, ZID T, Boukassoula $\mathrm{H}$, Ben Salah N. Situation et Politique en matiere de drogues 2014 [updated 2016/01/16/17:07:03]. Available from:http://www.ofdt.fr/BDD/publications/docs / Worbook2015PolitiqueStrategieNationale.pdf.

7. Ben Mosbah H. Etude de l'usage détourné et du sevrage à la Buprénorphine chez les toxicomanes admis en 2013 au centre d'Aide et D'Ecoute de l'Association Tunisienne de Luttecontre la Toxicomanie [Thesis]. Monastir: Faculty of Pharmacy of Monastir; 2014.

8. Tompkins DA, Smith MT, Mintzer MZ, Campbell CM, Strain EC. A double blind, within subject comparison of spontaneous opioid withdrawal from buprenorphine versus morphine. The Journal of pharmacology and experimental therapeutics. 2014;348(2):217-26.

9. Tripathi B, Hemaraj P, Dhar N. Buprenorphine withdrawal syndrome. Indian journal of psychiatry. 1995;37(1):23. 10. Jasinski DR, Pevnick JS, Griffith JD. Human pharmacology and abuse potential of the analgesic buprenorphine: a potential agent for treating narcotic addiction. Archives of General Psychiatry. 1978;35(4):501-16.

11. Wesson DR, Ling W. The clinical opiate withdrawal scale (COWS). Journal of psychoactive drugs. 2003;35(2):2539.

12. BECHEIKH D, GHACHEM R, ZALILA H, BOUSSET'TA A. Mésusage de buprénorphine haut dosage: Un phénomène émergent en Tunisie. Alcoologie et addictologie. 2008;30(1):25-8.

13. Uosukainen H, Ilomäki J, Kauhanen J, Tacke U, Föhr J, Tiihonen J, et al. Factors associated with buprenorphine compared to amphetamine abuse among clients seeking treatment in Finland. Journal of substance abuse treatment. 2014;46(5):561-6.

14. Fischer B, Nakamura N, Rush B, Rehm J, Urbanos- ki K. Changes in and characteristics of admissions to treatment related to problematic prescription opioid use in Ontario, 2004-2009. Drug and alcohol dependence. 2010;109(1):257-60.

15. Foster B, Twycross R, Mihalyo M, Wilcock A. Buprenorphine. Journal of pain and symptom management. 2013;45(5):939-49.

16. Brown SM, Holtzman M, Kim T, Kharasch ED. Buprenorphine metabolites, buprenorphine-3-glucuronide and norbuprenorphine-3-glucuronide, are biologically active. Anesthesiology. 2011;115(6):1251 PubMed .

17. Greenwald M, Johanson CE, Bueller J, Chang Y, Moody DE, Kilbourn M, et al. Buprenorphine duration of action: mu-opioid receptor availability and pharmacokinetic andbehavioral indices. Biological psychiatry. 2007;61(1):101-10.

18. Jaunin-Stalder N, Mazzocato C. Hommes et femmes: sommes-nous tous égaux face à la douleur? Revue médicale suisse. 2012;8(348):1470-3. PubMed

19. Moody DE, Fang WB, Morrison J, McCance-Katz E. Gender differences in pharmacokinetics of maintenance dosed buprenorphine. Drug and alcohol dependence. 2011;118(2):479-83.

20. Greenwald MK, Comer SD, Fiellin DA. Buprenorphine maintenance and mu-opioid receptor availability in the treatment of opioid use disorder: implications for clinical use and policy. Drug and alcohol dependence. 2014;144:1-11.

21. Barrio G, De La Fuente L, Lew C, Royuela L, Bravo MaJ, Torrens M. Differences in severity of heroin dependence by route of administration: the importance of length of heroin use. Drug and alcohol dependence. 2001;63(2):169-77.

22. Boscarino JA, Rukstalis M, Hoffman SN, Han JJ, Erlich PM, Gerhard GS, et al. Risk factors for drug dependence among out-patients on opioid therapy in a large US health-care system. Addiction. 2010;105(10):1776-82. PubMed

23. Hartwell KJ, Tolliver BK, Brady KT. Biologic commonalities between mental illness and addiction. Primary psychiatry. 2009;16(8):33 PubMed .

24. Dijkstra B, Krabbe P, De Jong C, van der Staak C. Prediction of withdrawal symptoms during opioid detoxification. J Opioid Manag. 2008;4(5):311-9. PubMed 25. Lofwall MR, Walsh SL. A review of buprenorphine diversion and misuse: the current evidence base and experiences from around the world. Journal of addiction medicine. 2014;8(5):315-26.

African Health Sciences Vol 16 Issue 4, December, 2016 
26. Häkkinen M, Launiainen T, Vuori E, Ojanperä I. Benzodiazepines and alcohol are associated with cases of fatal buprenorphine poisoning. European journal of clinical pharmacology. 2012;68(3):301-9.

27. Lintzeris N, Nielsen S. Benzodiazepines, methadone and buprenorphine: interactions and clinical management. The American Journal on Addictions. 2010;19(1):59-72. 28. vanDorp E, Yassen A, Sarton E, Romberg R, Olofsen E, Teppema L, et al. Naloxone reversal of buprenorphine-induced respiratory depression. Anesthesiology. 2006;105(1):51-7.

29. Hermann D, Klages E, Welzel H, Mann K, Croissant B. Low efficacy of non-opioid drugs in opioid withdrawal symptoms. Addiction biology. 2005;10(2):165-9. PubMed 30. Epstein DH, Preston KL. No evidence for reduction of opioid-withdrawal symptoms by cannabis smoking during a methadone dose taper. The American Journal on Addictions. 2015;24(4):323-8.
31. de Wet C, Reed L, Glasper A, Moran P, Bearn J, Gossop M. Benzodiazepine co-dependence exacerbates the opiate withdrawal syndrome. Drug and alcohol dependence. 2004;76(1):31-5.

32. Bruce RD, Govindasamy S, Sylla L, Kamarulzaman A, Altice FL. Lack of reduction in buprenorphine injection after introduction of co-formulated buprenorphine/ naloxone to the Malaysian market. The American journal of drug and alcohol abuse. 2009;35(2):68-72.

33. Ahmadi J, Ahmadi K. Controlled trial of maintenance treatment of intravenous buprenorphine dependence. Irish journal of medical science. 2003;172(4):171-3.

34. Rolland B, Muyssen A, Danel T, Cottencin O. Faut-il retirer la buprénorphine haut-dosage du marché français ? Revue d'Épidémiologie et de Santé Publique. 2013;61(2):91-2. PubMed

35. World-Health-Organisation. WHO Expert Committee on Drug Dependence: Thirty-third report. Geneva: World Health Organization; 2003. p. 1-31. 